\title{
Design and Implementation of Fire Extinguisher Robot with Robotic Arm
}

\author{
Abdul Waris Memon ${ }^{1}$, Juan $\mathrm{Du}^{1}$, Abdul Haleem Abro $^{2}$, Sharmeen Iftikhar Shah ${ }^{2}$ and Moazzam Ali Bhutto ${ }^{3}$ \\ ${ }^{1}$ School of Automation Science and Engineering, South China University of Technology, China \\ ${ }^{2}$ Department of Electronic-Information Technology, University of Sindh Jamshoro, Sindh, Pakistan \\ ${ }^{3}$ School of Electronic Information and Engineering, South China University of Technology, China
}

\begin{abstract}
Robot is a device, which performs human task or behave like a human-being. It needs expertise skills and complex programming to design. For designing a fire fighter robot, many sensors and motors were used. User firstly send robot to an affected area, to get live image of the field with the help of mobile camera via Wi-Fi using IP camera application to laptop. If any signs of fire shown in image, user direct robot in that particular direction for confirmation. Fire sensor and temperature sensor detects and measures the reading, after confirmation robot sprinkle water on affected field. During extinguish process if any obstacle comes in between the prototype and the affected area the ultrasonic sensor detects the obstacle, in response the robotic arm moves to pick and place that obstacle to another location for clearing the path. Meanwhile if any poisonous gas is present, the gas sensor detects and indicates by making alarm.
\end{abstract}

\section{Introduction}

\subsection{Fire Extinguisher Robot}

A fire extinguisher robot is one that have a fire quencher included to it. By attaching a fire quencher of the robot, the mechanization put off the fire of the affected area. This paper comprises those outline and development of a robot that is able to detect and quench fire. This robot executes the fire extinguish and fire detection idea. It has utilization of fire sensors which detect the affected fired area. A robot put off the recreated tunnel fire, industry, school, home and forests fire. Flame sensors are used for the initial detection of the fire, If the flame is detected then the robot resonances those alerts with the help of ringer which is set on the prototype, the robot actuates an electronic valve discharging sprinkles of water on the fired area. This project helps to develop interests as well as innovations in the fields of robotics while working towards a practical and obtainable solution to save lives and diminish the risk of property destruction. This fire detector robot will play its role, where human fails to detect fire. Fire contenders face dangerous circumstances when smothering blazes furthermore rescuing victims, it may be an unavoidable part and condition for a fire warrior. Done contrast, a robot has capacity and functioned by itself or be controlled from a distance. In short, fire extinguisher robots have decline the need to fire contenders should get under hazardous circumstances [1-2].

\subsection{Robotic Arm}

This robot also implements removing of obstacles with a robotic arm. Robotic arm is used to pick and place the obstacles from one place to another location. It is used to clear the path and to reach the affected area easily. Obstacles are detected by ultrasonic sensor.

\section{Literature Review}

\subsection{Fire Extinguisher Robot}

Home could furnish safety, convenience, and productivity for men in the $21 \mathrm{st}$ century. Several functions, frameworks and systems are used to integrate an intelligent home system. A standout amongst the practically significant frameworks may be the fire identification work framework in a smart home system. In the previous literatures, huge numbers masters Look into in the administration robot. Exactly Look into tended to done Creating target-tracking framework for administration robot [3-5], for example, Hisato Kobayashi et al. Recommended a strategy on identify individual towards a self-sufficient versatile watch robot [6]. Moreover, exactly scrutinize tended to in the robot need the ability about fire-fighting. Ruser, furthermore Magori depicted those fire identification with a consolidation for ultrasonic also microwave doppler sensor. Ruser, H. Furthermore Magori, V, "Fire identification with joined together ultrasonic-microwave doppler sensor," in incidents from claiming IEEE ultrasonic Symposium, Vol. 1, 1998, pp. 489-492. Luo 
also $\mathrm{Su}$ use two smoke sensors, two temperature sensors and two fire sensors to identify fired affected area, and finding which sensor will be disappointment utilizing versatile combination strategy.

\subsection{Robotic Arm}

Robotic manipulators also robotic arms were those exceptionally first robots which were utilized within the industries and they have been in used since 1960s. An extensive number for investigations need centered once robots for making a difference people with physical challenges, to example, those desktop robotic framework Toward Hammel. With the proceeded advancement in registering power, machine vision, Furthermore propelled sensors, researches need began to concentrate on employments for robotic arms over Every day an aggregation Also fill in situations in one task for example, Care-O-bot and the late versatile controller robot PR2 starting with Willow carport. Drug store assignments incorporated capacity oversaw economy from claiming medication and taking care of risky solution.

\section{Methodology}

Fire extinguisher robot with a robotic arm is divided in to three sections. Such as- Obstacle Detection, Temperature, Flame and Gas Sensors Detection Robot Direction Control.

\subsection{Obstacle Detection}

The block diagram, flow chart and schematic diagram of obstacle detection are shown in Fig 1, Fig 2 and Fig 3 in which ultrasonic sensor senses the obstacle and sends signal to the arduino (1), arduino generates the command for the robotic arm to clear the path.

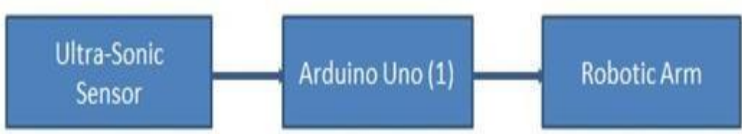

Figure 1. Block Diagram of Obstacle Detection

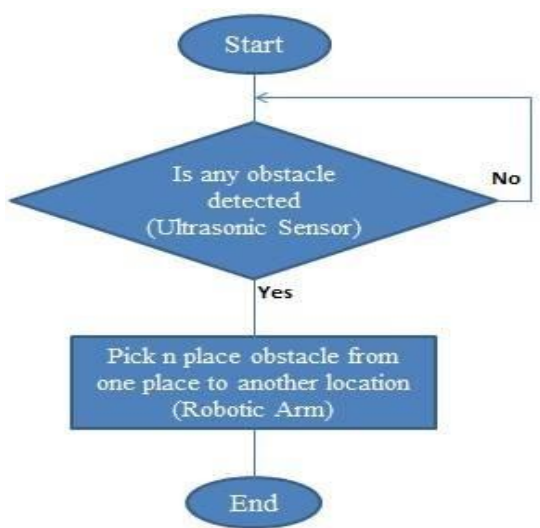

Figure 2. Flow Chart of Obstacle Detection

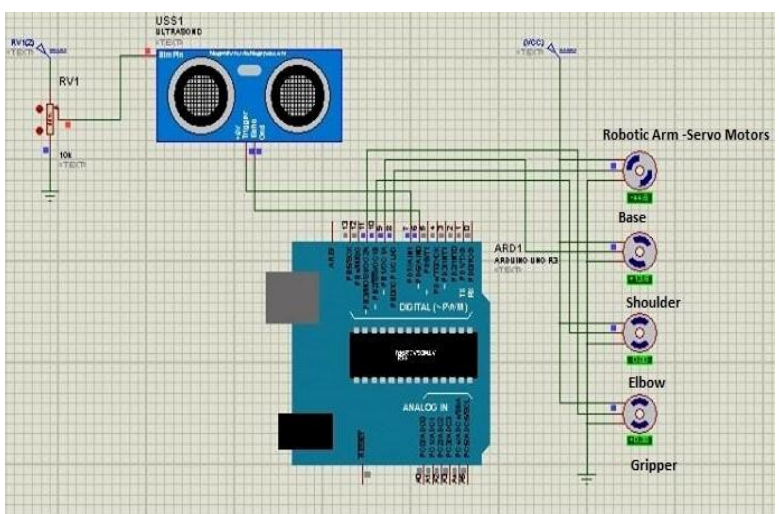

Figure 3. Schematic Diagram of Obstacle Detection

\subsection{Temperature, Flame and Gas Sensors Detection}

The block diagram and flow chart of flame, gas, and temperature sensors detection are shown in Figure 4 and Figure 5 in which temperature sensor senses the temperature of environment, if the temperature crosses its limit, it activates the flame sensor to sense the fire. Further, flame sensor sends the signal to arduino (2). After that, arduino generates the command for water sprinkler to make the fire off from the desired location.

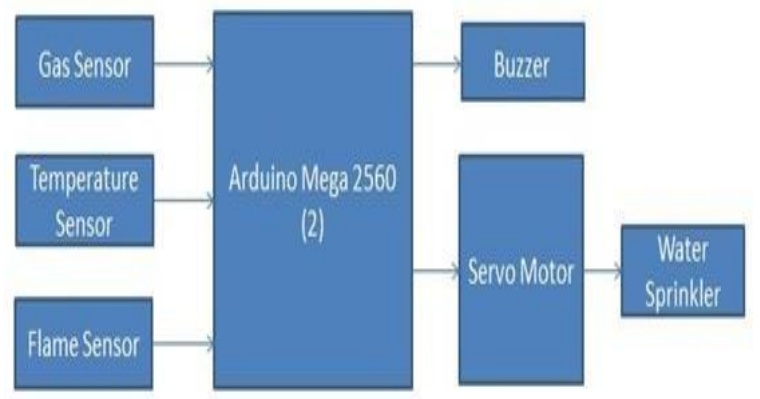

Figure 4. Block Diagram of Temperature, Flame and Gas Sensors Detection
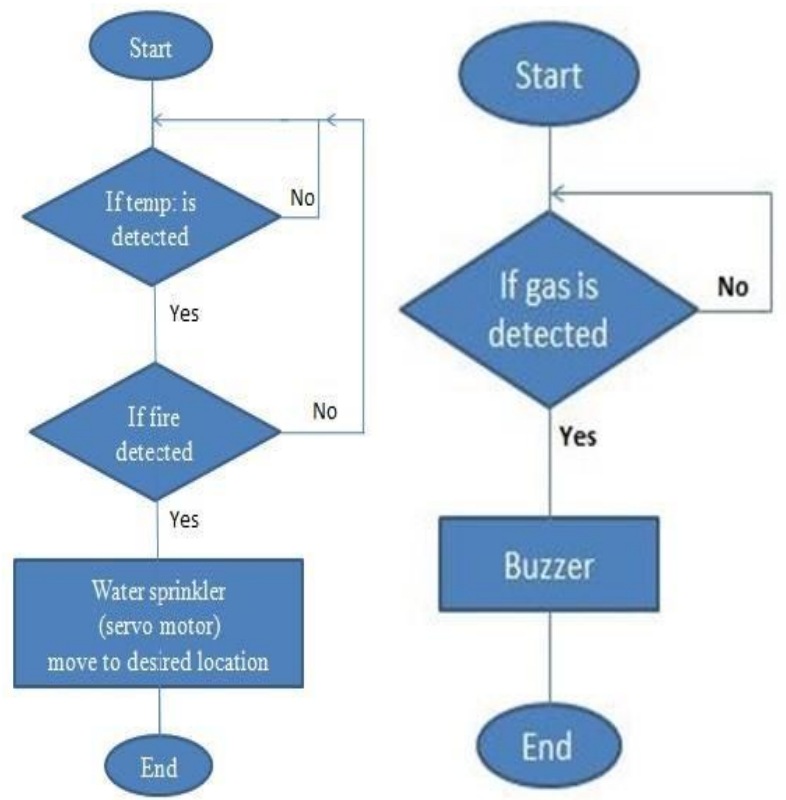

Figure 5. Flowchart of Temperature Sensor Detection and Gas Sensor Detection 


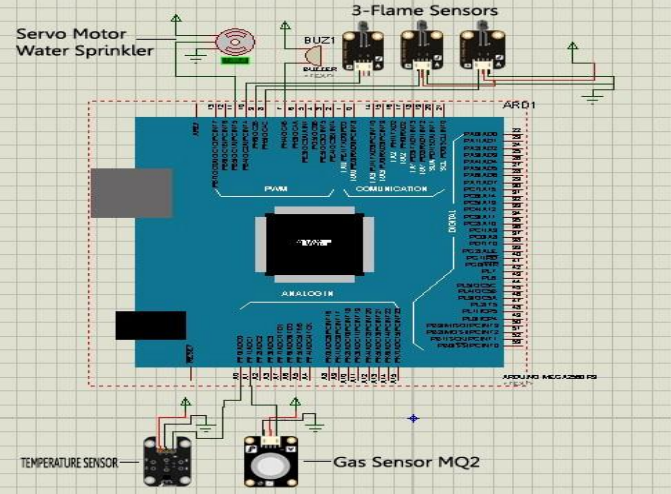

Figure 6. Schematic Diagram of Gas, Temperature Sensor Detection

\subsection{Robot Direction Control}

The block diagrams of video feed backing and robot movement are shown in Fig 7 (a) and Fig 7 (b) in which android cell phone is connected to the laptop via mobile IP camera application which is connected with its IP address of desired location. Further, RF remote control controls the robot direction through it, robotic car can be moved to any direction.

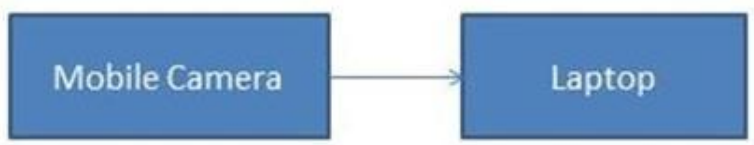

Wi-Fi

Figure 7(a). Block Diagram of Video Feed Backing

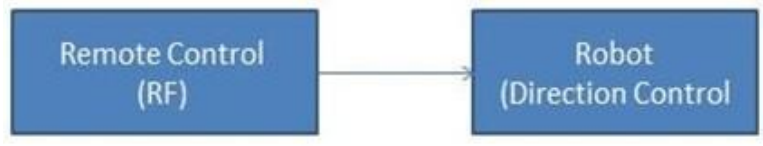

Figure 7(b). Block diagram of Robot Movement

\section{Results}

\subsection{Obstacle Detection}

The process of obstacle detection is shown in figure 8 to figure 12, picking and placing the obstacle towards left side for path clearing.

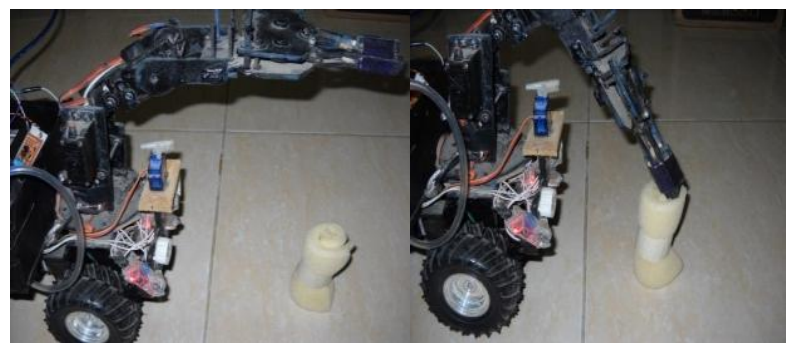

Figure 8. In this figure prototype is Detecting the obstacle \& Robotic Arm is moving towards Obstacle

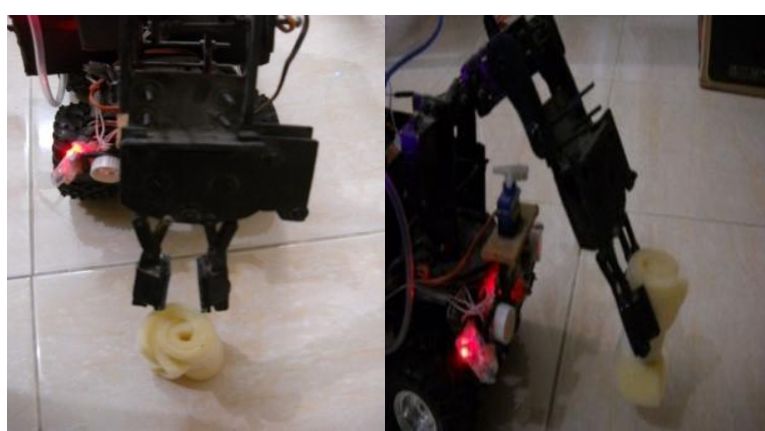

Figure 9. Robotic Arm is Opening the Gripper \& Robotic Arm is picking the Obstacle

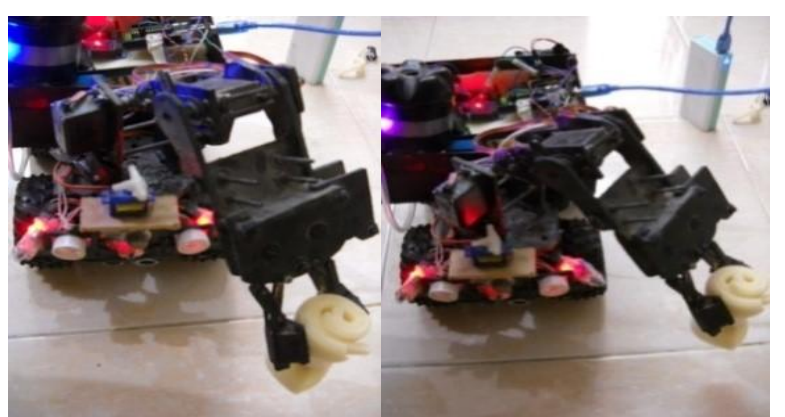

Figure 10. Robotic Arm is moving the obstacle at Left Side to clear its path

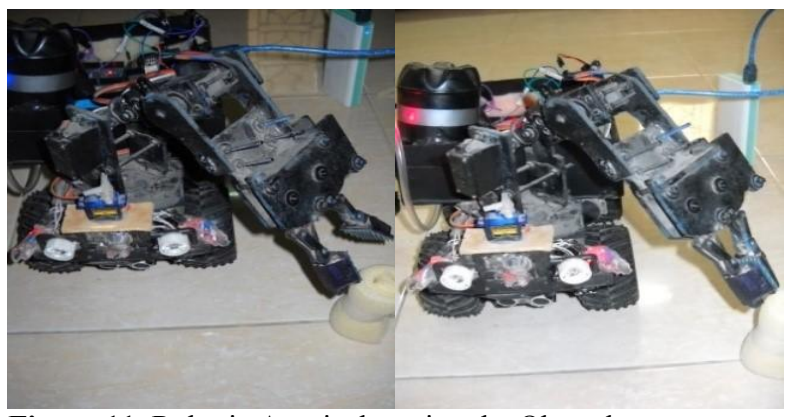

Figure 11. Robotic Arm is dropping the Obstacle

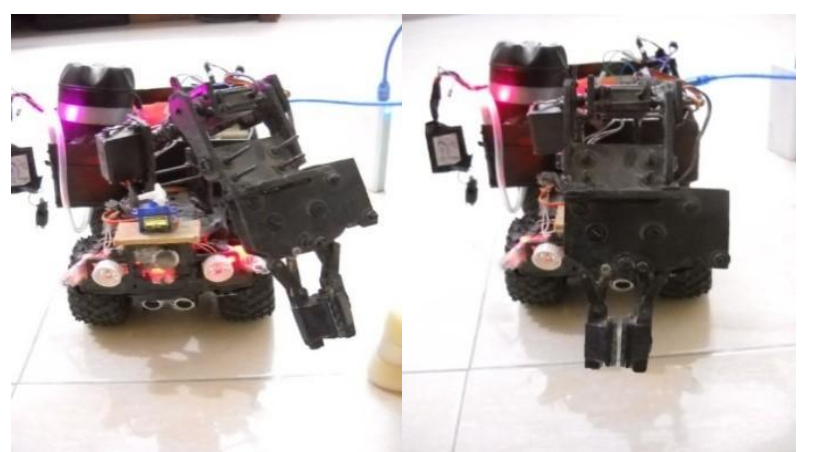

Figure 12. Finally in this image Robotic Arm is closing the Grip \& Robotic Arm is coming towards Initial Position

\subsection{Temperature, Flame and Gas Sensor Detection}

\subsubsection{Temperature Sensor Detection}

Figure 13 shows the Temperature Sensor Detection. The robot is in sleep mode. It will be switched on if any temperature can be detected by the temperature sensor. 


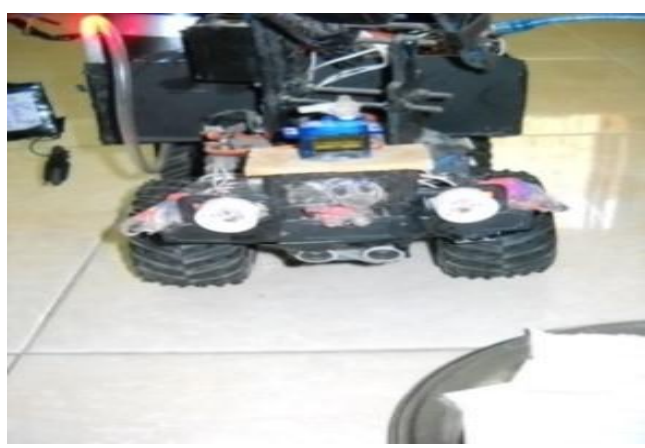

Figure 13. Temperature Sensor Detection

\subsubsection{Flame Sensor Detection}

The fire sensing process is illustrated from Figure 14 to 16 , Figure 14 shows that fire has been detected on left side and extinguished by the water sprinkler. Figure 15 shows that fire is detected at center and extinguished by water sprinkler. Figure 16 shows that fire is detected at right side and extinguished by water sprinkler. When the water sprinkler extinguishes the fire the pump will be stopped automatically.

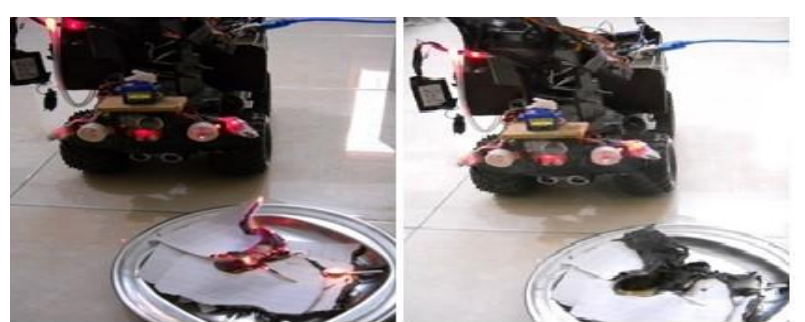

Figure 14. Fire Sensing At Left Side and Fire Extinguished By the Water Sprinkler

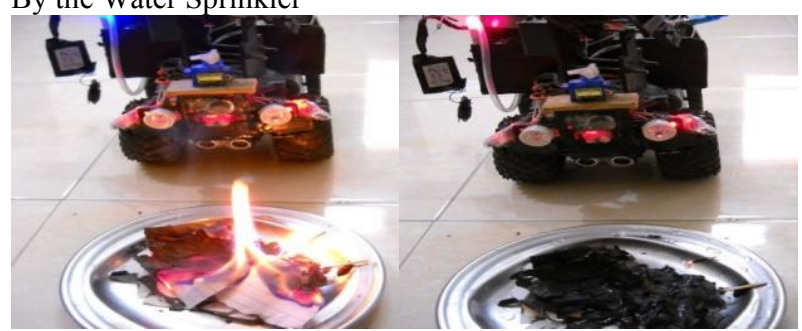

Figure 15. Fire Sensing At Centre \& Fire Extinguished By the Water Sprinkler

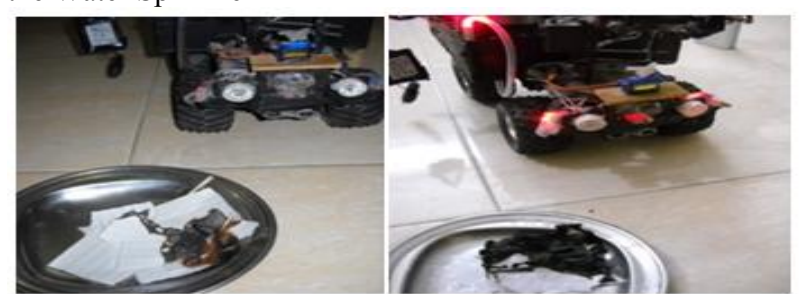

Figure 16. Fire Sensing At Right Side \& Fire Extinguished By the Water Sprinkler

\subsubsection{Gas Sensor Detection}

Figure 17 shows the detection of gas by gas sensor from $10 \mathrm{~cm}$ and $15 \mathrm{~cm}$ and beep the buzzer to indicate the presence of poisonous gas.

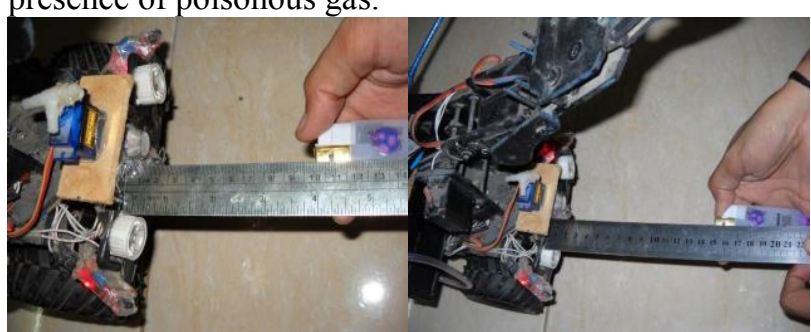

Figure 17. Detection of Gas $10 \mathrm{~cm} \&$ Detection of Gas $15 \mathrm{~cm}$

\subsubsection{Robot Direction Control}

An IP camera application is used for video feed-backing, which is shown in Figures 18 to Figure 19. An IP Camera application is used through android cell phone on transmission side and in resulting visualization of location is received on monitoring side.
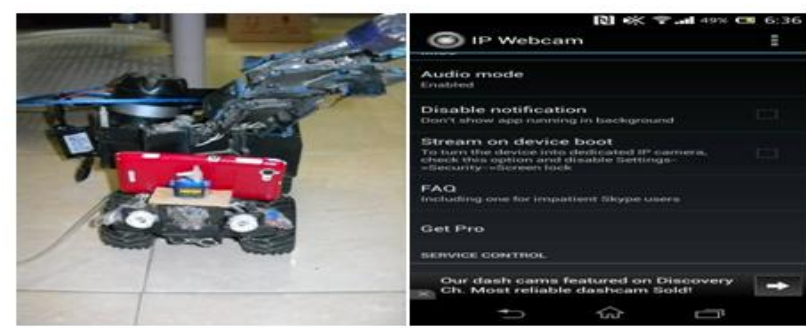

Figure 18. Prototype with IP Camera \& IP Webcam Cell Phone Application

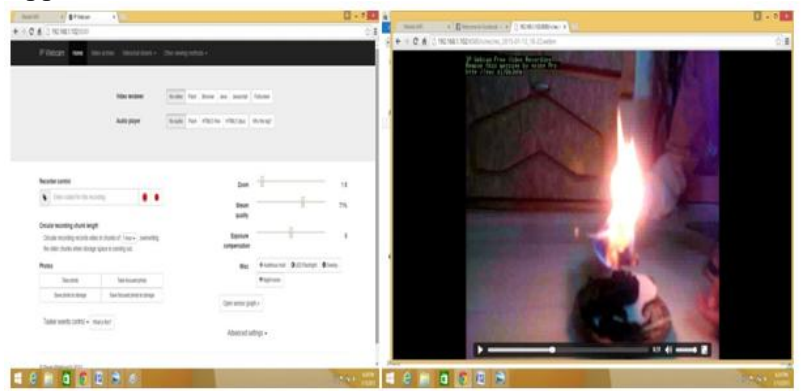

Figure 19. IP Camera on Transmitter \& Receiver Side (Laptop) and Video Feed-backing On Receiving Side

\section{Conclusion}

Fire-fighting robot can be easily and conveniently used and operated automatically when any fire incident occurs in educational, industrial and hospital areas to save human life. Fire-fighting Robot comprises of numerous sensors and motors, and has small in size, less in weight, with rechargeable batteries, in result it requires less space. Prototype provides us greater efficiency to detect the flame, temperature and gas presented in the affected area. The extinguisher robot effectively extinguishes fire before it becomes uncontrollable and gives threat to life. Fire-fighting robot also successfully removes obstacle from the path to another location by using a robotic arm to clear the way. Hence, the robot can play a crucial role in our society. 


\section{References}

1. Et. Akshay Desmukh, "A survey on fire detector and extinguisher robot," (5th International Conference on Recent Development in Engineering Science, Humanities and Management, pp. 256-264, 16th April 2017), ISBN : 978-93-86171-39-9.

2. Et. Dr. Raju N.Pamnchal, "Fire Fighting Vehicle," International Journal of Engineering Science and Research (IJETSR)," Volume 4, (Issue 9, September 2017), ISSN 2394-3386.

3. Et. Ting-Li Chien, "Detection system of security robot using multisensor fusion algorithms," The Fourteenth International Symposium on Artificial Life and Robotics 2009(AROB 14th 09), " February 5-7, 2009.

4. Et. S.O. Lee, "A stable target-tracking control for unicyle mobile robots," Proceedings of the IEEE/RSJ International Conference on Intelligent Robots and Systems(IROS 2000)," Vol.3, 2000, pp. 1822-1827.

5. L. E. Parker and B. A. Emmons, "Cooperative multirobot observation of multiple moving targets," Proceedings of the IEEE/RSJ International Conference on Robotics and Automation," Vol.3, 1997, pp. 2082-2089.

6. Et. Kuo L. Su, "Design a low cost security robot applying in family," 2nd International Conference on Autonomous Robots and Agents, (December 13-15, 2004).

\footnotetext{
Project Supported by: (1) Fundamental Research Funds for the Central Universities (Grant No. 2015ZM135) ; (2) Science and Technology Project of Nansha, Guangzhou, Guangdong (Grant No. 2015KF012) ; (3) Science and Technology Project of Guangzhou (Grant No. 201707010061); (4) Science and Technology Project (International Cooperation Research) of Nansha, Guangzhou, Guangdong (Grant No. 2016GJ012).
} 